\title{
Symmetrical generalization decrements: Configural stimulus processing in human contingency learning
}

\author{
Anna Thorwart and Harald Lachnit \\ Philipps-Universität Marburg, Marburg, Germany
}

\begin{abstract}
Models of associative learning differ in their predictions concerning the symmetry of generalization decrements. Whereas Pearce's (1994) configural model predicts the same response decrement after adding elements to and after removing elements from a previously trained stimulus, elemental models, such as the replaced elements model and Harris's (2006) model, anticipate more of a decrement for removing than for adding elements. In three contingency learning experiments, we manipulated the motion and the spatial arrangement of colored dots in order to induce configural or elemental processing by perceptual grouping. The results reliably showed symmetrical decrements for the added and removed groups. The manipulations of the stimuli had no effect on stimulus processing. This is in line with Pearce's configural model, but it is at variance with the elemental models and previous studies.
\end{abstract}

Stimulus generalization occurs when a response to a new and neutral stimulus resembles a conditioned response (CR) to a previous conditioned stimulus (CS). After eating a poisonous apple, we presumably will experience nausea not only if we taste the same kind of apple again, but also if we taste a similar kind of apple. Knowledge about the $\mathrm{CS}$ - the poisonous apple - generalizes to a new stimulus - the other apple. Generalization between stimuli is a vital ability because it allows for probably appropriate responding to new stimuli. Responding to the new stimulus, however, will normally be weaker than responding to the trained stimulus. This difference in response strength is referred to as generalization decrement.

Models of associative learning explain generalized responding to an apparently new stimulus by assuming that this new stimulus will share components ${ }^{1}$ with the previously conditioned CS. This is true for so-called elemental and configural models. Although previous research has often tried to ascertain whether processing is configural or elemental, the issue has now shifted toward identifying the conditions under which processing is best described by configural theories and those under which it is best described by elemental theories (Melchers, Shanks, \& Lachnit, 2008, with commentaries; Shanks, Lachnit, \& Melchers, 2008). Distinctions among associative learning models are based on differences in how these models conceptualize the coding, processing, and representation of stimuli. In configural models like Pearce's (1987, 1994), a stimulus compound is represented and associated as a whole. Generalization is based on the coactivation of representations of similar compounds, with the amount of coactivation depending on the proportion of common components. The central characteristic of elemental models, on the other hand, is the assumption that individual components of a stimulus compound are learned about. Stimuli with common components will therefore activate the same associations. Modern elemental models, such as the replaced elements model (REM; Wagner, 2003) and Harris's (2006) model, assume flexible nonlinearity, which means that a compound need not activate exactly the same associations as its components. ${ }^{2}$

In the following studies, we compared the application of configural models and of elemental models to two different generalization tasks. In the first task, the new stimulus (test stimulus) was created by removing some components of the just-trained CS (e.g., training AB + and testing A). This course of action is equivalent to the design of overshadowing experiments. In the second task, after training the CS (e.g., A+), some new components are added (e.g., $\mathrm{AB})$. If responding to the test stimulus $\mathrm{AB}$ is weaker than responding to the initially trained $\mathrm{A}$, this can be described as external inhibition. Both overshadowing and external inhibition are well-known phenomena in the study of associative learning (Pavlov, 1927).

We simulated the predictions of the different models by using several MATLAB-based programs (Schultheis, Thorwart, \& Lachnit, 2008a, 2008b; Thorwart, Schultheis, König, \& Lachnit, in press). Pearce's (1994) configural model predicts symmetrical decrements; that is, the amount of decrement produced by testing $\mathrm{AB}$ after training with $\mathrm{A}$ will be the same as that produced by testing $\mathrm{A}$ after training with AB. The decrement is based on the coactivation of similar configural units, and just as similarity is symmetric, so too are generalization decrements. On the

A. Thorwart, anna.thorwart@staff.uni-marburg.de 
other hand, both elemental theories predict asymmetrical generalization decrements: Removal of components always results in a larger decrement than does adding them. Interestingly, the flexibility of the elemental models under consideration does not allow us to predict symmetrical decrements. In sum, comparing generalization decrements after adding and after removing components theoretically clears the way for contrasting the predictions of Pearce's (1994) configural model with those of Wagner's (2003) and Harris's (2006) elemental models.

Up to now, studies that have investigated generalization decrement explicitly have routinely observed asymmetrical generalization decrements (Brandon, Vogel, \& Wagner, 2000; Glautier, 2004; González, Quinn, \& Fanselow, 2003; Wheeler, Amundson, \& Miller, 2006). Brandon et al. trained rabbits in an eyelid-conditioning study with $\mathrm{A}+, \mathrm{AB}+$, or $\mathrm{ABC}+$. Using cross-modal stimuli (light, tone, vibration) and testing with $\mathrm{A}, \mathrm{AB}$, and $\mathrm{ABC}$, Brandon et al. observed larger generalization decrements after removing than after adding a stimulus component. González et al. conditioned rats across 2 consecutive days. A single unsignaled footshock was presented in the presence of specific contextual cues (light, tone, odor). Using freezing as a measure of conditioning, removal - but not addition — of a stimulus component of the training context produced a significant generalization decrement. Glautier (2004) and Wheeler et al. used causal learning tasks to investigate generalization decrements. In Glautier's (2004) experiments, participants had to rate the amount of air pollution produced by airplanes. Stimuli created by adding novel features (such as markings or guns) to the previously trained airplanes were rated at the same level as the original training stimuli, but stimuli created by removing features led to reduced ratings. Wheeler et al., in a food-poisoning scenario, observed that adding a labeled picture of food to a previously learned picture of food can produce a generalization decrement, but removing produces a more robust decrement. Although these studies differed in the size of decrements produced by adding a stimulus component, they nevertheless converged in the observation that removing stimulus components produces larger decrements. Therefore, all of these authors concluded that elemental models were better than configural ones in describing stimulus processing during stimulus generalization.

In spite of these findings, there may yet be circumstances in which configural processing operates. For other associative learning tasks, such as summation or blocking, it has been shown that results consistent with both configural and elemental accounts can be obtained (Melchers et al., 2008). In an attempt to investigate whether manipulation of the stimuli is able to produce symmetrical generalization decrements, we addressed the question, "Under which conditions is processing best described by configural theories, and under which conditions is it best described by elemental theories?" One successful attempt in this line concerned the spatial arrangement of the stimulus components in blocking experiments (Glautier, 2002). Blocking was reduced when the same components were presented in close proximity, whereas spatial separation of the components enhanced blocking.
A common explanation relates perceptual grouping and gestalt principles to associative learning. The term perceptual grouping describes the notion that various elements in a complex display may be perceived as "going together" (Palmer, 1999). It seems reasonable that objects seen as belonging together perceptually may be processed configurally. Thus, the manipulation of perceptual grouping of the components may affect processing in associative learning. According to the gestalt principle of proximity, objects that are closer together will be seen as belonging together (Palmer, 1999). This would explain the results of Glautier (2002). If this is the case, other gestalt principles too should have a comparable impact on stimulus processing and, by this, on the symmetry of generalization decrements.

Common fate is another powerful principle, according to which objects moving with the same speed in the same direction become perceptually grouped and are seen as belonging together (Palmer, 1999). Therefore, we used this principle in an attempt to induce configural processing in a stimulus-generalization study.

\section{EXPERIMENT 1}

In the present experiment, small dots differing in color were seen moving across a black screen for $2 \mathrm{sec}$. The kind of motion varied as a dichotomous between-participants factor: In the separated motion conditions, the direction of motion was randomly chosen for each dot and trial, with some restrictions; in the grouped motion conditions, all dots were moving in the same direction, chosen randomly for each trial. To compare the decrements, the second between-participants factor task was included: Added groups were trained with two stimulus configurations (one reinforced and the other nonreinforced), consisting of two different-colored dots each. Thus, color was the relevant feature, whereas motion was irrelevant for the outcome. For removed groups, the two training stimuli (one reinforced and the other nonreinforced) consisted of five different-colored dots each. In the test stage after training, the five- and the two-dot compounds were presented in both tasks. This two-factor design resulted in four experimental groups: separated-added, separated-removed, grouped-added, and grouped-removed (see Table 1). The qualitative patterns of the predictions already have been described above: If perceptual grouping can induce configural processing, generalization decrements should be asymmetrical in the separated condition, but symmetrical in the grouped condition.

\section{Method}

Participants. We recruited 140 participants from the PhilippsUniversität Marburg campus by posted advertisement and by face-toface contact. They received course credits or were paid with sweets. We excluded 44 participants from further analysis because they did not solve the discrimination at the end of the training. To be included, the rating of the reinforced stimulus had to be higher than 4 , and that of the nonreinforced stimulus had to be lower than 4 , in the last trial of training; these numbers refer to scores on a 7-point scale (see below). The remaining 96 participants were from 18 to 48 years of age $(M=23.69)$. Sixty-nine women took part. We tested 20, 25, 30, and 21 participants in the grouped-removed, separated-removed, grouped-added, and separated-added groups, respectively. 
Table 1

Design of Experiments 1, 2, and 3

\begin{tabular}{|c|c|c|c|c|c|}
\hline \multirow[b]{2}{*}{ Experiment } & \multicolumn{2}{|c|}{ Group } & \multirow[b]{2}{*}{ Training Stage } & \multicolumn{2}{|r|}{ Test Stage } \\
\hline & Stimuli & Task & & Trained Stimuli & Test Stimuli \\
\hline \multirow[t]{2}{*}{1} & $\begin{array}{l}\text { separated } \\
\text { grouped }\end{array}$ & added & $\mathrm{AB}+, \mathrm{QR}-$ & $\mathrm{AB}, \mathrm{QR}$ & ABCDE, QRSTU \\
\hline & $\begin{array}{l}\text { separated } \\
\text { grouped }\end{array}$ & removed & $\mathrm{ABCDE}+, \mathrm{QRSTU}-$ & ABCDE, QRSTU & $\mathrm{AB}, \mathrm{BC}, \mathrm{CD}, \mathrm{DE}, \mathrm{AE}, \mathrm{QR}, \mathrm{RS}, \mathrm{ST}, \mathrm{TU}, \mathrm{QU}$ \\
\hline 2 & - & $\begin{array}{l}\text { added } \\
\text { removed }\end{array}$ & $\begin{array}{l}\mathrm{AB}+, \mathrm{QR}- \\
\mathrm{ABC}+, \mathrm{QRS}-\end{array}$ & $\begin{array}{l}\mathrm{AB}, \mathrm{QR} \\
\mathrm{ABC}, \mathrm{QRS}\end{array}$ & $\begin{array}{l}\mathrm{ABC}, \mathrm{QRS} \\
\mathrm{AB}, \mathrm{BC}, \mathrm{AC}, \mathrm{AB}, \mathrm{AC}, \mathrm{QR}, \mathrm{RS}, \mathrm{QS}, \mathrm{QR}, \mathrm{RS}\end{array}$ \\
\hline 3 & $\begin{array}{l}\text { variable } \\
\text { fixed }\end{array}$ & (within-subjects) & $\mathrm{AB}+, \mathrm{CD}-, \mathrm{EFG}+, \mathrm{HIJ}-$ & $\mathrm{AB}, \mathrm{CD}, \mathrm{EFG}, \mathrm{HIJ}$ & ABX, CDY, EF, IJ \\
\hline
\end{tabular}

Note-Each letter refers to a dot with a specific color. A "+" indicates that the temperature was above the critical limit (reinforced trials), and a "-" indicates that the temperature was below the critical limit (nonreinforced trials). The numbers of trials are not specified (see text for details).

Apparatus and Stimuli. The experiment took place either in our psychology labs or in the reading room of the university's student services house. In the labs, the stimuli were presented on 15-in. color monitors with $1,024 \times 768$ resolution and a $75-\mathrm{Hz}$ refresh rate. In the student services house, an IBM ThinkPad was used, placed in a paperboard cabin. The monitor was 15 in., with $1,024 \times 768$ resolution and a $60-\mathrm{Hz}$ refresh rate. The participants wore soundattenuating earmuffs.

Different-colored dots were used as the CS. The size of the dots was 16 pixels. Ten different colors were chosen (RGB: 64, 204, 255; $53,169,55 ; 234,50,24 ; 147,36,207 ; 238,29,90 ; 187,212,65$; $231,95,217 ; 69,49,181 ; 255,157,0 ; 253,228,111)$. The colors were assigned randomly for each participant but, overall, the separated and grouped conditions were matched in the colors used.

The dots moved across a central $600 \times 600$ pixel area with a speed of 2 pixels/frame. One trial comprised 150 frames. The starting points and trajectories of the dots were chosen randomly for each participant and each trial. The only restriction was that the distance between the dots had to be greater than 25 pixels at any time during movement. In the separated conditions, one trajectory for each dot was chosen randomly per trial, whereas in the grouped conditions, one trajectory common for all dots per trial was computed. The background was black during the whole experiment.

Stimulus generation and experimental control was accomplished with MATLAB 7, using the Psychophysics Toolbox 2.54 (Brainard, 1997).

Procedure. At the start of the experiment, each participant was shown the following instructions (in German) on the screen:

Dear participant! Thank you for your readiness to participate in this experiment.

Please imagine that you are working in the control center of an atomic power plant. A visual display that reflects the temperature of the cooling water is located on your control desk. Unfortunately, the computer that normally evaluates whether the temperature reflected in the visual display has crossed a critical limit or not is broken. Your task in this experiment is to replace this computer.

For that purpose, the visual display is presented initially. Please look at it carefully. Then you are asked to guess whether the temperature has crossed the critical limit or not. Perhaps you will not be perfectly sure with your decision; therefore, a 7-point answer scale is available, ranging from 1 (definitely not) to 7 (definitely). At the beginning, you will have to guess. After a while, however, the decision will become easier as, after you deliver your rating, you will receive feedback whether the temperature had actually crossed the critical limit or not. For all of your answers, accuracy, rather than speed, is essential. Please do not take notes during the experiment. If you have any more questions, please ask them now. If you don't have any questions, please start the experiment by clicking on the "next" button.
When a participant asked a question, it was answered by the experimenter. When the participant clicked on the "next" button, the learning stage began with the first CS. Then the dots disappeared, and the question "Has the temperature crossed the critical limit?" was shown, along with a 7-point rating scale, with the anchors marked definitely not and definitely. After the participants marked one of the check boxes, they received feedback indicating either that the temperature was above the critical limit (in reinforced trials) or that it was below the critical limit (in nonreinforced trials). In the test stage, this feedback was omitted. Before the test stage, additional instructions were shown:

In the following part of the experiment, we would like to survey what you have learned. For that purpose, there will be additional, new visual displays, but no feedback. Please look carefully at the visual display and enter your rating.

The training stage consisted of 15 reinforced and 15 nonreinforced trials. For the two added groups, this was $\mathrm{AB}+$ and $\mathrm{QR}-$; in the case of the two removed groups, it was ABCDE+ and QRSTU- (with different letters indicating different-colored dots and $+/-$ indicating reinforced and nonreinforced trials). In the test stage, five trials of $\mathrm{AB}, \mathrm{ABCDE}, \mathrm{QR}$, and $\mathrm{QRSTU}$ were displayed in the added groups. The removed groups were tested with $\mathrm{AB}, \mathrm{BC}, \mathrm{CD}, \mathrm{DE}, \mathrm{AE}, \mathrm{QR}$, RS, ST, TU, QU, and five trials of ABCDE and QRSTU each. Due to an experimental error, the first 6 participants did not receive the stimuli related to the nonreinforced trained stimulus in the test stage. However, since the training was the same, their test trials related to the reinforced trained stimuli were included in the analyses. All trial sequences were randomized but were kept parallel for the separated and the grouped conditions.

\section{Results}

For all statistical analyses, an $\alpha$ level of .05 and the Huynh-Feldt correction for the degrees of freedom was used.

Figure 1A shows that the rating of the reinforced and the nonreinforced stimuli diverged during training. In the last training trials, the mean ratings were $6.68(S D=$ $0.657)$ and $1.26(S D=0.603)$. The training was analyzed with a $2 \times 15 \times 2 \times 2$ ANOVA that included the withinparticipants factors contingency (reinforced vs. nonreinforced) and trial (1-15) and the between-participants factors task (added vs. removed) and motion (separated vs. grouped). As expected, the main effect of contingency $\left[F(14,92)=271.61, p<.001, \eta_{\mathrm{p}}^{2}=.75\right]$, the main effect of trial $\left[F(14,1288)=4.05, p<.001, \eta_{\mathrm{p}}^{2}=.04\right]$, and the contingency $\times$ trial interaction $[F(14,1288)=36.07, p<$ $\left..001, \eta_{\mathrm{p}}^{2}=.28\right]$ were significant. All other $F$ s were below 

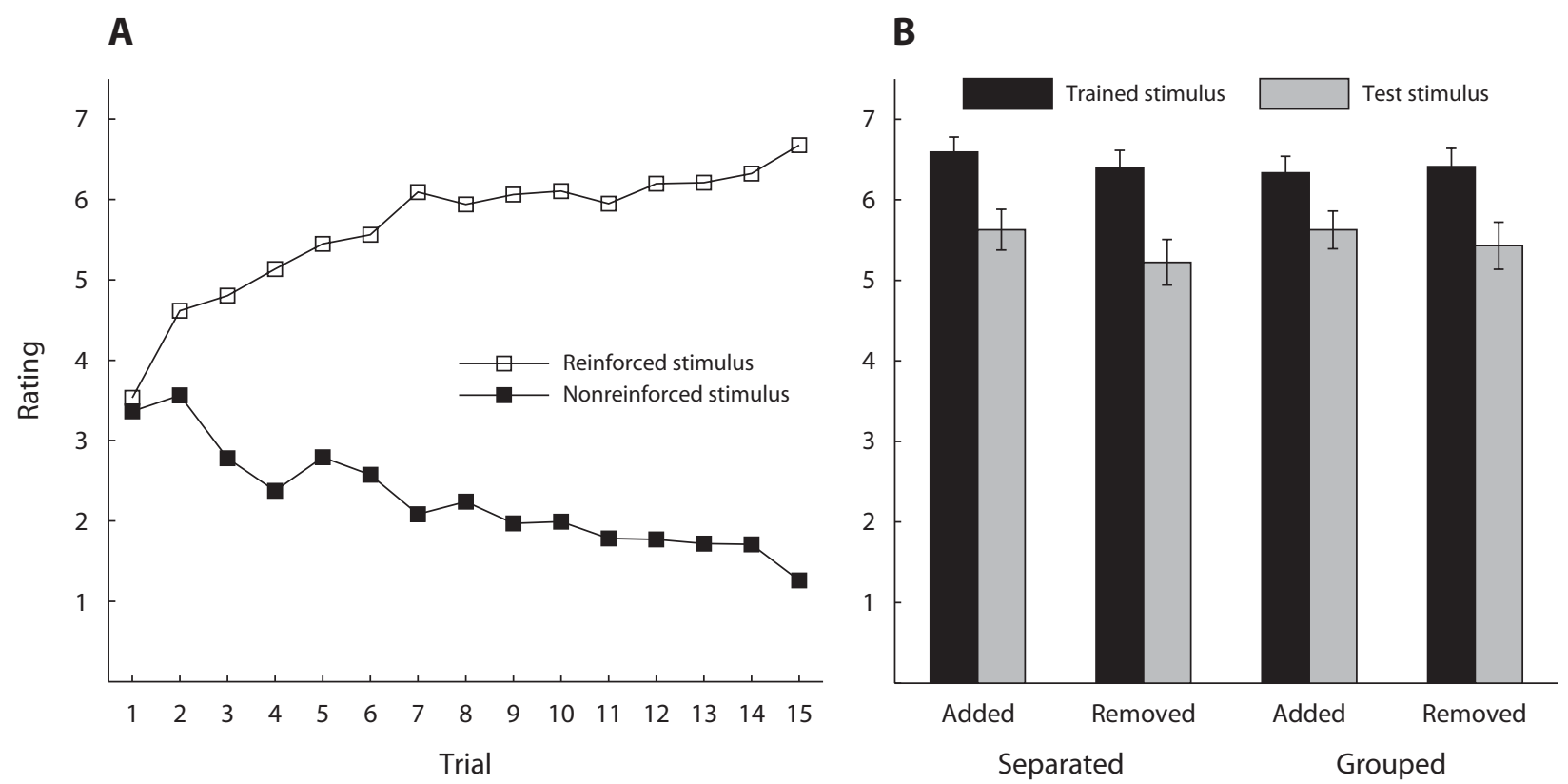

Figure 1. Mean ratings from 1 (definitely not) to 7 (definitely) in Experiment 1. Panel A shows ratings in the training stage, averaged across groups for the reinforced $(\mathrm{AB}+/ \mathrm{ABCDE}+)$ and nonreinforced $(\mathrm{QR}-/ \mathrm{QRSTU}-)$ stimulus compounds. Panel $\mathrm{B}$ displays, separately for the four experimental groups, the mean ratings of the reinforced trained stimulus and the corresponding test stimulus in the test stage.

$1.70\left(\right.$ all $p \mathrm{~s}>.07$, all $\eta_{\mathrm{p}}^{2} \mathrm{~s}<.02$ ). Thus, independent of the task or the kind of motion, the discrimination was learned successfully.

Figure 1B displays the mean ratings in the test stage for the stimuli related to the reinforced trained stimulus. The black bars represent the rating of the previously trained stimulus itself, whereas the gray bars represent the rating of the corresponding test stimulus. In the added groups, for example, the rating of $\mathrm{AB}$ is shown by the black bar and the rating of $\mathrm{ABCDE}$ by the gray bar. The test stimulus was rated lower than the reinforced CS in all groups, and the decrement looks similar for all groups. To analyze the test stage, a $2 \times 5 \times 2 \times 2$ ANOVA was computed, including the within-participants factors cue (trained vs. test) and trial (1-5) and the between-participants factors task (added vs. removed) and motion (separated vs. grouped). Only the main effect of cue was significant $[F(1,92)=$ $67.00, p<.001, \eta_{\mathrm{p}}^{2}=.43$ ] (all other $F_{\mathrm{s}}<1.47, p \mathrm{~s}>$ $\left..21, \eta_{\mathrm{p}}^{2} \mathrm{~s}<.02\right)$. Thus, there was a generalization decrement in all groups. Neither the kind of task nor the kind of motion had any influence on the size of generalization decrements. Comparing the generalization tasks, $\eta_{\mathrm{p}}^{2}$ for the main effect of cue was .47 in the removed groups and .39 in the added groups.

Although the nonreinforced stimuli were introduced to the experimental design only as filler cues (because the theories do not differ in their predictions), their results are reported for the sake of completeness: The mean rating for the nonreinforced trained stimulus $(\mathrm{QR}-$ for the added groups and QRSTU - for the removed groups) was 1.66 $(S D=1.27)$. The mean rating for the corresponding test stimulus was $2.7(S D=1.44)$. Thus, the newly introduced test stimuli were rated higher than the nonreinforced trained stimuli. We conducted a $2 \times 5 \times 2 \times 2$ ANOVA with the within-participants factors cue (trained vs. test) and trial (1-5) and the between-participants factors task (added vs. removed) and motion (separated vs. grouped). There was a significant main effect of cue $[F(1,86)=$ 44.02, $\left.p<.001, \eta_{\mathrm{p}}^{2}=.339\right]$ and a significant cue $\times$ motion $\times$ trial interaction $\left[F(1,86)=4.22, p<.05, \eta_{\mathrm{p}}^{2}=\right.$ $.047]$. All other effects were not significant $\left(F_{\mathrm{S}}<2.25\right.$, $\left.p \mathrm{~s}>.06, \eta_{\mathrm{p}}^{2} \mathrm{~s}<.025\right)$.

\section{Discussion}

About two thirds of the participants successfully mastered the discrimination. Analyzing only "learners" ensured that the reinforced stimuli were rated high enough to provide the opportunity for observing decrements in the test. (Wheeler et al., 2006, have shown that failing to observe a decrement at all is often due to effects that are too small.) The test stimuli were rated lower than the reinforced training stimulus but above neutral; therefore, a generalization decrement was observed in all four groups. Comparing the added and removed groups, all decrements were of the same size. This symmetry is in line only with Pearce's configural model. Neither the REM nor Harris's (2006) model can account for these results. Thus, we have to conclude that the stimuli were processed configurally in both perceptual grouping conditions. The decrements were already symmetrical in the separated conditions; an additional grouping effect of motion in the grouped conditions was unobservable due to a floor effect.

Therefore, it is impossible to draw conclusions about the relationship of perceptual grouping and configural processing from the results of Experiment 1. Something must have influenced stimulus processing in Experiment 1, 
because previous studies have consistently reported asymmetrical decrements (Brandon et al., 2000; Glautier, 2002; González et al., 2003; Wheeler et al., 2006). Although these studies differed in paradigms, stimuli, species, and experimental settings, they always observed asymmetry of generalization decrement. Therefore, one would expect that asymmetrical decrements are a quite robust phenomenon. Thus, the question arises: What distinguishes Experiment 1 from all these studies?

\section{EXPERIMENT 2}

A first difference between our Experiment 1 findings and those of other studies might be related to the motion of the stimuli. Normally, stimuli are presented at fixed positions during one trial. Motion per se may have had a configural impact in Experiment 1. Palmer (1999) described the new principle of synchrony as being related to the classical principle of common fate, in the sense that it is a dynamic factor, but different in that the "fate" of the elements does not have to be common, as long as the change occurs at the same time. Thus, the common feature of motion might have been enough to perceptually group the dots, even if each dot moved in a different direction. In the second experiment, we therefore "froze" the colored dots on their starting positions; the dots were presented at the starting positions, without movement.

\section{Method}

The materials and procedure in Experiment 2 were identical to those in Experiment 1, with the following exceptions.

Participants. We recruited 60 participants from the PhilippsUniversität Marburg campus by posted advertisement and by faceto-face contact. We excluded 15 participants from further analysis be- cause they did not solve the discrimination at the end of the training. The remaining 45 participants were from 18 to 39 years of age $(M=$ 22.93). Thirty-four women took part. Twenty-one participants were tested in the added group, and 24 were tested in the removed group.

Stimuli. The different-colored dots were the same as those used in Experiment 1, but there was no motion. The position of the dots in the $600 \times 600$ pixel area in the center of the screen was chosen randomly for each participant and each trial. There was a minimal distance of 25 pixels between adjacent dots. The dots were shown for 150 frames, as before, but did not move.

Procedure. The training stage comprised 15 reinforced and 15 nonreinforced trials. The number of removed and added dots was reduced to 1, since the effects in Experiment 1 were large and the discrimination should be easier with three, instead of five, dots. The added group had to learn $\mathrm{AB}+$ versus $\mathrm{QR}-$, and the removed group had to learn $\mathrm{ABC}+$ versus $\mathrm{QRS}-$. Again, the trial sequences were randomized for each participant. In the test stage, $\mathrm{AB}, \mathrm{ABC}, \mathrm{QR}$, and QRS were presented five times each in the added group, and the removed group was tested with $\mathrm{AB}, \mathrm{BC}, \mathrm{AC}, \mathrm{AB}, \mathrm{AC}, \mathrm{QR}, \mathrm{RS}, \mathrm{QS}$, $\mathrm{QR}, \mathrm{RS}$, and five trials of $\mathrm{ABC}$ and $\mathrm{QRS}$ each.

\section{Results and Discussion}

Figure $2 \mathrm{~A}$ shows the mean rating during training. In the last trial, the mean rating for the reinforced stimulus was $6.84(S D=0.475)$ and, for the nonreinforced stimulus, 1.11 $(S D=0.383)$. The training scores were analyzed with a $2 \times$ $15 \times 2$ ANOVA with the within-participants factors contingency (reinforced vs. nonreinforced) and trial (1-15) and the between-participants factor task (added vs. removed). The main effect of contingency $[F(14,43)=603.99, p<$ $\left..001, \eta_{\mathrm{p}}^{2}=.94\right]$ and the main effect of trial $[F(14,602)=$ $\left.2.38, p<.02, \eta_{\mathrm{p}}^{2}=.05\right]$ were significant. Furthermore, the contingency $\times$ trial interaction $[F(14,602)=48.49, p<$ $\left..001, \eta_{\mathrm{p}}^{2}=.53\right]$ and the triple interaction contingency $\times$ trial $\times \operatorname{task}\left[F(14,602)=2.06, p<.05, \eta_{\mathrm{p}}^{2}=.05\right]$ were significant (all other $F \mathrm{~s}<1$ ). The significant triple interac-
A

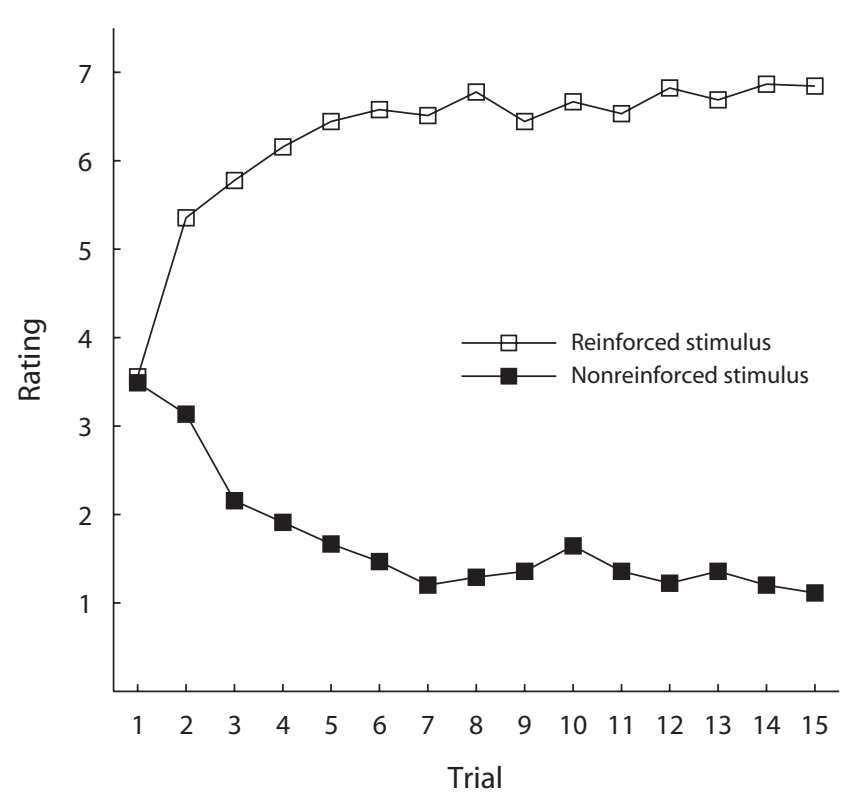

B

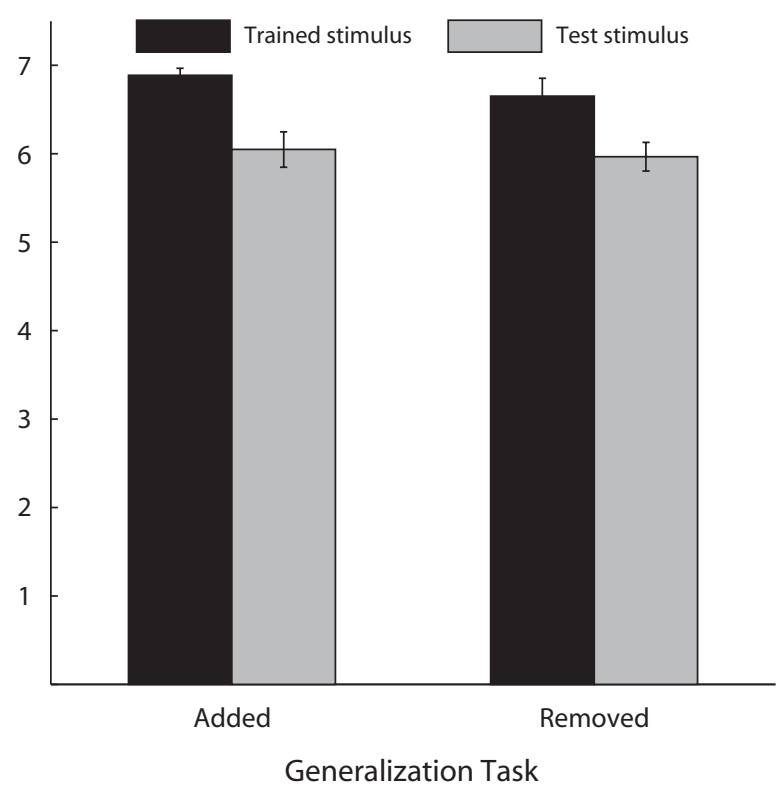

Figure 2. Mean ratings from 1 (definitely not) to 7 (definitely) in Experiment 2. Panel A shows ratings in the training stage, averaged across groups for the reinforced $(\mathrm{AB}+/ \mathrm{ABC}+)$ and nonreinforced $(\mathrm{QR}-/ \mathrm{QRS}-$ ) stimulus compounds. Panel $\mathrm{B}$ displays, separately for two "generalization tasks," the mean ratings of the reinforced trained stimulus and the corresponding test stimulus in the test stage. 
tion was probably due to differences in the first trial. On average, the added group rated the nonreinforced stimulus higher than the reinforced stimulus, whereas the removed group showed the reverse.

Figure 2B shows the mean ratings in the test stage of Experiment 2. For the removed group, the trained stimulus was $\mathrm{ABC}$, and the test stimuli were $\mathrm{AB}, \mathrm{BC}$, and $\mathrm{AC}$. For the added group, the trained stimulus was $\mathrm{AB}$, and the test stimulus was $\mathrm{ABC}$. The rating of the test stimuli was smaller than the rating of the trained stimuli in both groups.

To analyze the test stage, a $2 \times 5 \times 2$ ANOVA was computed with the within-participants factors cue (trained vs. test) and trial (1-5) and the between-participants factor task (added vs. removed). Only the main effect of cue was significant $\left[F(1,43)=33.06, p<.001, \eta_{\mathrm{p}}^{2}=.44\right]$ (all other $\left.F \mathrm{~s}<1.23, p \mathrm{~s}>.28, \eta_{\mathrm{p}}^{2} \mathrm{~s}<.03\right)$. Thus, there was a generalization decrement in all groups. The kind of task had no influence on the amount of generalization decrement. Comparing the main effect of cue in the added and removed tasks, $\eta_{\mathrm{p}}^{2}$ was .46 for the removed group and .42 for the added group.

The mean rating for the nonreinforced trained stimulus $(\mathrm{QR}$ - for the added groups and QRS - for the removed groups) was $1.28(S D=0.83)$. The mean rating for the corresponding test stimulus was $1.96(S D=0.89)$. Thus, the newly introduced test stimuli were rated higher than the nonreinforced trained stimuli. We conducted an ANOVA with the within-participants factors cue (trained vs. test) and trial (1-5) and the between-participants factors task (added vs. removed). There was a significant main effect of cue $\left[F(1,43)=31.25, p<.001, \eta_{\mathrm{p}}^{2}=.421\right]$. All other effects were not significant $\left(F s<2.19, p \mathrm{~s}>.12, \eta_{\mathrm{p}}^{2} \mathrm{~s}<\right.$ $.048)$.

The discrimination was mastered by about $75 \%$ of the participants. It is clear that it was not the motion of the dots that induced configural processing in Experiment 1, because the results of Experiment 2 were quite similar. This replicates the results of Experiment 1 but is still at variance with the previous studies. In order to enhance the comparability of the present study with these previous studies, we presented the stimuli on a fixed position in the middle of the screen in a third experiment.

\section{EXPERIMENT 3}

In contrast to previous studies, there were no fixed positions for the stimulus components in Experiment 2. Instead, the dots were randomly distributed across more or less the entire screen. This may have enhanced the impression of the whole screen as one stimulus and, thus, the different dots might have been processed as a whole. Therefore, the following experiment included a betweenparticipants factor of position. In the variable group, the dots were randomly positioned as in Experiment 2; in the fixed group, the dots were presented on three selected positions in the middle of the screen. Furthermore, Experiment 2 revealed that adding and removing one dot to a two-dot compound was enough to generate stable decrements. This allowed us to use a within-participants design for the generalization task. All participants now received four stimulus compounds during training - two compounds with two dots and two with three dots, one reinforced each - and eight compounds in test — four with two and four with three dots (see Table 1). This resulted in 12 different colors needed for the whole experiment, instead of the 20 different colors that would have been needed in a case of within-participants design and four five-dot compounds in Experiment 1.

\section{Method}

The materials and procedure in Experiment 3 were the same as those used in Experiment 2, with the following exceptions.

Participants. Forty-one of 63 participants successfully solved the discrimination during training and were included in the analyses. They were from 18 to 29 years of age $(M=21.27)$. Thirty-one women took part. Nineteen participants were tested with variableand 22 with fixed-dot positions.

Stimuli. Two additional colors (RGB: 0, 0, 224; 200, 255, 200) were defined, resulting in 12 different colors. Stimulus generation in the variable group was the same as in Experiment 2. In the fixed group, the same number and colors of dots were used, but the dots were presented at three fixed positions during the whole experiment. The positions were chosen so that the distance between them was equal to the mean distance of the dots in the variable group and that the dots were presented on a horizontal line in the middle of the screen. The colored dots were randomly assigned to a position in each trial.

Procedure. There were some slight modifications of the instructions, for clarification. Unlike in Experiments 1 and 2, the factor task was manipulated within participants. The training stage comprised 15 trials of $\mathrm{AB}+, \mathrm{CD}-, \mathrm{EFG}+$, and $\mathrm{HIJ}-$. In test, $\mathrm{AB}, \mathrm{CD}, \mathrm{EFG}$, $\mathrm{HIJ}, \mathrm{ABX}, \mathrm{CDY}, \mathrm{EF}$, and IJ were presented twice each.

\section{Results and Discussion}

Figure $3 \mathrm{~A}$ shows the rating during training. In the last trial, the mean ratings for $\mathrm{AB}+, \mathrm{CD}-, \mathrm{EFG}+$, and $\mathrm{HIJ}-$ were $6.88(S D=0.400), 1.10(S D=0.374), 6.88(S D=$ $0.400)$, and $1.12(S D=0.400)$, respectively. The training stage was analyzed with a $2 \times 2 \times 15 \times 2$ ANOVA with the within-participants factors contingency (reinforced vs. nonreinforced), task (added vs. removed), and trial (1-15) and the between-participants factor group (variable vs. fixed). The main effect of contingency was significant $\left[F(14,39)=334.50, p<.001, \eta_{\mathrm{p}}^{2}=.90\right]$, as was the contingency $\times$ trial interaction $[F(14,546)=40, p<.001$, $\left.\eta_{\mathrm{p}}^{2}=.51\right]\left(\right.$ all other $\left.F \mathrm{~s}<3, p \mathrm{~s}>.10, \eta_{\mathrm{p}}^{2} \mathrm{~s}<.07\right)$.

Figure $3 \mathrm{~B}$ shows the mean ratings in the test stage of Experiment 3 . The ratings of the test stimuli $\mathrm{ABX}$ and $\mathrm{EF}$ were smaller than the ratings of the trained stimuli $\mathrm{AB}$ and $\mathrm{EFG}$. To analyze the test stage, a $2 \times 2 \times 5 \times 2$ ANOVA was computed with the within-participants factors cue (trained vs. test), task (added vs. removed), and trial ( 1 or 2 ) and the between-participants factor group (variable vs. fixed). Only the main effect of cue was significant $[F(1,92)=$ $36.89, p<.001, \eta_{\mathrm{p}}^{2}=.49$ ] (all other $F \mathrm{~s}<2.78, p \mathrm{~s}>.10$, $\eta_{\mathrm{p}}^{2} \mathrm{~s}<.07$ ). Thus, there was a generalization decrement in all groups. Neither the kind of task nor the kind of spatial arrangement had an influence on the amount of generalization decrement. Comparing the size of the main effects of cue in the added and the removed tasks, $\eta_{\mathrm{p}}^{2}$ was .329 for $\mathrm{AB}$ versus $A B X$ and .437 for EFG versus EF. 

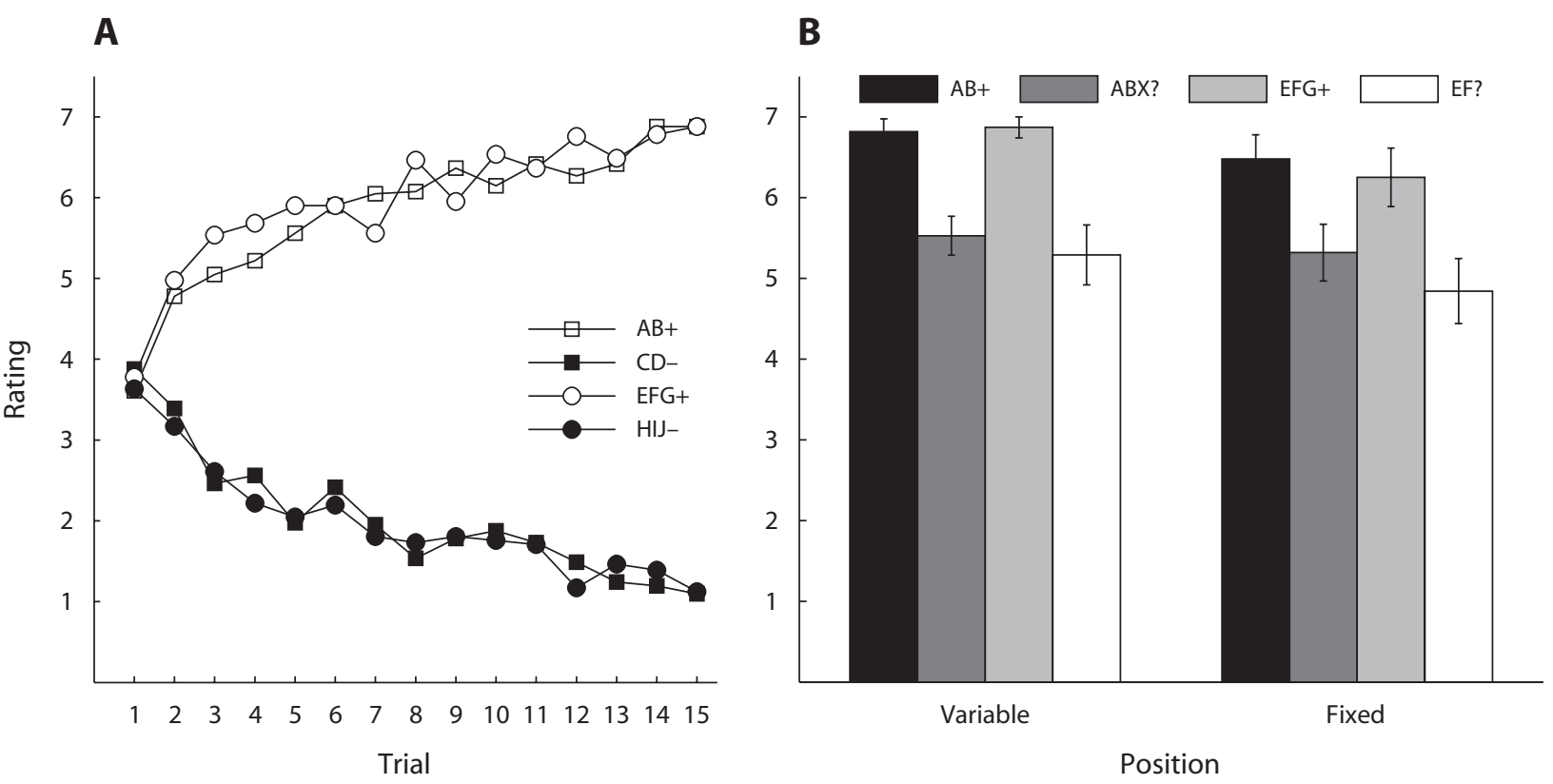

Figure 3. Mean ratings from 1 (definitely not) to 7 (definitely) in Experiment 3. Panel A shows ratings in the training stage, averaged across groups for the two reinforced and two nonreinforced stimulus compounds. Panel B displays, separately for the manipulation of the stimulus position, the mean ratings of the reinforced trained stimuli $(\mathrm{AB}+/ \mathbf{E F G}+)$ and the corresponding test stimuli $(\mathrm{ABX}$ ?/EF?) in the test stage.

The mean ratings for $\mathrm{CD}$, HIJ, CDY, and $\mathrm{HI}$ were 1.34 $(S D=0.83), 1.41(S D=1.02), 3.11(S D=1.77)$, and $2.96(S D=1.79)$, respectively. Thus, the newly introduced test stimuli were rated higher than the nonreinforced trained stimuli. We conducted an ANOVA with the withinparticipants factors cue (trained vs. test), task (added vs. removed), and trial (1 or 2) and the between-participants factor group (fixed vs. variable). There was a significant main effect of cue $\left[F(1,39)=55.76, p<.001, \eta_{\mathrm{p}}^{2}=.588\right]$ and a significant four-way interaction $[F(1,39)=4.11$, $\left.p<.05, \eta_{\mathrm{p}}^{2}=.095\right]$. All other effects were not significant $\left(F \mathrm{~s}<3.07, p \mathrm{~s}>.09, \eta_{\mathrm{p}}^{2}<.07\right)$.

The results of Experiment 3 replicated the results of Experiments 1 and 2. About two thirds of the participants mastered the discrimination, and we found a stable decrement for the added and the removed generalization task in both groups. All decrements were of about the same size. This means that the presentation of the dots on fixed positions in the middle of the screen did not help to produce asymmetrical decrements, as observed in previous experiments in other labs.

\section{GENERAL DISCUSSION}

We compared generalization decrement in two different generalization tasks in three experiments. In the added task, a new stimulus was added to the previously trained $\mathrm{CS}$, whereas in the removed task, a stimulus was removed from the previously trained CS. Experiments 1, 2, and 3, all using colored dots in a power-plant scenario (but different kinds of stimulus arrangements), resulted in symmetrical generalization decrements. This is the first time that symmetrical decrements have been reliably observed in studies investigating generalization decrements. This symmetry is predicted by Pearce's (1994) configural model, whereas Wagner's (2003) and Harris's (2006) elemental models both predict asymmetrical generalization decrements.

Before discussing potential factors controlling stimulus processing, and before explaining the divergence from the results of previous studies, we have to exclude an alternative explanation for the observed symmetrical decrements. In each of the discriminations of Experiments 1, 2, and 3, there was no need to rely on all the components presented as stimuli. Theoretically, the discriminations could have been solved by using only one component (one colored dot). If indeed the participants had relied on only one component (for example, the most salient one), removing "irrelevant" components would have led to no decrement at all. On the other hand, the decrement after removing the "relevant" component should have been at maximum, since the stimulus that carried all the associative strength was no longer present at test. Therefore, elemental models still predict that removing a stimulus should result in more of a decrement on average than adding a stimulus, even if only part of the stimulus compound is used to solve the discrimination. Nevertheless, future studies of generalization decrements should address this issue more directlyfor example, by assessing whether all single stimuli are rated alike.

The results of Experiments 1, 2, and 3 suggest that the stimuli were processed configurally and that this processing was not influenced by the kind of motion, the motion per se, or the spatial arrangement of the stimuli, although other studies have shown an impact of the kind of stimulus presentation (e.g., Glautier, 2002; Livesey \& Boakes, 
2004). Furthermore, the spatial arrangement in the fixed group of Experiment 3 was similar to that of Wheeler et al.'s (2006) Experiment 2, and both experiments used clearly separable visual stimuli. But for all of that, the results differ. What in the power-plant experiments was so powerful in inducing configural processing that omitting other potential manipulations, such as the common motion of the dots, did not result in elemental processing? Three factors come to mind in comparing these three experiments with previous studies-especially the causal learning studies of Wheeler et al. and Glautier (2004).

A first hypothesis concerns within-trial timing. From other studies in our lab comparing causal learning and classical conditioning, we have clues that the amount and pressure of time may influence stimulus processing. Lachnit, Schultheis, König, Üngör, and Melchers (2008) and Lachnit, Thorwart, and Schultheis (2008) observed different kinds of stimulus processing in different paradigms and response systems. They used modified positive and negative patterning designs with two different kinds of visual stimuli (pictures of foods, colored rectangles) in eyelid-conditioning, skin-conductance-conditioning, and causal-learning experiments. The kind of stimuli had no impact on stimulus processing. Instead, systematic differences were observed, depending on the response system and the time for stimulus processing. Pearce's configural model was best in predicting early stimulus processing in skin conductance conditioning, whereas elemental models were superior in causal-learning studies in which stimulus duration was paced by the participants. In conditioning experiments with fixed interstimulus intervals (ISIs), there is a time limitation, and further results provided some hints that reducing the ISI (and thus enhancing time pressure) in an eyelid-conditioning study influenced learning toward configural processing. In sum, Pearce's configural model predicted early stimulus processing; other theories predicted late stimulus processing better. This factor might explain the results of our present experiments. In the power-plant studies, stimulus presentation was limited to $2 \mathrm{sec}$, whereas the ISI in Wheeler et al.'s (2006) experiments was paced by the participants. Thus, in human contingency learning with time pressure, correct predictions are made by Pearce's configural theory and, without time pressure, the predictions of elemental theories are correct. Hence, reducing the time pressure in the power-plant scenario should result in asymmetrical decrements. Glautier's (2004) results contradict this explanation: His planes traversed the screen in approximately 2.1 to $2.8 \mathrm{sec}$, and he found no decrement for the added stimuli. However, Wheeler et al. have already suggested alternative explanations for Glautier's (2004) results. Furthermore, since there was no need to respond during training in Glautier's paradigm, the issue of time pressure on stimulus processing may not have been relevant for learning.

Second, there is a further difference among the scenarios used in the different studies. Wheeler et al.'s (2006) foodpoisoning scenario, as well as Glautier's (2004) air pollution scenarios, are causal scenarios. The participants had to rate the likelihood that the meals would cause food poisoning or how much pollution was caused by an airplane, respectively. In the power-plant scenario of the present experiments, no statement was made about the causal relationship between the dots and the cooling water's temperature. Instead, it was only said that there is a visual display that reflects the temperature of the cooling water and that the participants should predict whether the temperature had crossed the critical limit. De Houwer, Beckers, and Glautier (2002) have shown that blocking was stronger when the cues were presented as potential causes of the outcome rather than as mere indicators or predictors of the outcome. In the causal conditions of De Houwer et al.'s experiments, the participants were asked to rate the likelihood that firing a weapon would be followed by the destruction of a tank (i.e., the outcome), whereas in the predictive conditions, they were asked to rate the likelihood of the destruction of the tank when an abstract visual figure was present. Pineño, Denniston, Beckers, Matute, and Miller (2005) observed reliable overshadowing only between causes and only when the test question was causal. In order to study and contrast predictive and causal learning, they either used different wording in instructions about the relationship of two different stimuli and an outcome or took advantage of participants' preexperimental knowledge. Furthermore, test questions asked for the predictive and causal relationships separately. Therefore, the symmetry in our experiments may not have been due to a large decrement in the added groups but to reduced overshadowing (i.e., small decrements) in the removed groups in predictive contingency learning tasks. It requires the use of a causal scenario with moving dots to test this hypothesis.

Finally, it may be possible to provide an elemental explanation for the symmetrical decrements obtained here, in terms of generalization among stimulus components. Our discrimination tasks involved a large number of colors and, perhaps, there was generalization between single components (e.g., from a red dot to an orange dot). Furthermore, since the nonreinforced stimuli were rated well below the neutral level, it seems reasonable to assume generalization not only of excitation, but also of inhibition. If $\mathrm{C}, \mathrm{D}$, or $\mathrm{E}$ are similar in color to the nonreinforced $\mathrm{Q}$ or $\mathrm{R}$, this would reduce the prediction given for $\mathrm{ABCDE}$ after training $\mathrm{AB}+$. Thus, what has been interpreted as external inhibition could have been generalization of inhibition from the nonreinforced cues Q and R. Of course, there is no reason to expect a systematic generalization effect of this sort; colors were randomly allocated to conditions. However, if it occurred only in some proportion of participants, this may have been sufficient to produce the observed amount of response decrement to ABCDE. And although it is true that one would expect an equal similarity relationship between $\mathrm{C}, \mathrm{D}$, and $\mathrm{E}$ to $\mathrm{A}$ and $\mathrm{B}$, this might not produce complementary evidence for excitatory generalization for two reasons: First, ratings of the $\mathrm{AB}$ compounds were already at ceiling values (close to 7), which would have prevented any further increment in ratings by the participants for whom $\mathrm{C}, \mathrm{D}$, or $\mathrm{E}$ were similar to A and B. Second, the presence of A and B colors within the same test stimulus would presumably reduce generalization, because participants would more readily recognize that colors $\mathrm{C}, \mathrm{D}$, and $\mathrm{E}$ were not the same as $\mathrm{A}$ 
or B. As stated here, this argument relies on generalization from $\mathrm{Q}$ and $\mathrm{R}$ to $\mathrm{C}, \mathrm{D}$, or $\mathrm{E}$, due to a possible similarity in hue. A way to control this would be to use stimulus material that is less likely to support generalization between the components, as could be the case with labeled pictures of food (as Wheeler et al., 2006, used in their experiments) or, more relevant to the present study, with labels of colors.

\section{AUTHOR NOTE}

This research is part of the doctoral thesis of A.T. and was supported by the Deutsche Forschungsgemeinschaft graduate program NeuroAct (DFG 885). H.L. was supported by Grant La 564/19-1. We thank Steven Glautier for supporting A.T. in the course of her research visit to his lab in Southampton. Correspondence concerning this article should be addressed to A. Thorwart, Department of Psychology, Philipps-Universität Marburg, Gutenbergstraße 18, 35032 Marburg, Germany (e-mail: anna .thorwart@staff.uni-marburg.de).

\section{REFERENCES}

Brainard, D. H. (1997). The Psychophysics Toolbox. Spatial Vision, 10, 433-436.

Brandon, S. E., Vogel, E. H., \& Wagner, A. R. (2000). A componential view of configural cues in generalization and discrimination in Pavlovian conditioning. Behavioural Brain Research, 110, 67-72.

De Houwer, J., Beckers, T., \& Glautier, S. (2002). Outcome and cue properties modulate blocking. Quarterly Journal of Experimental Psychology, 55A, 965-985.

GlaUtier, S. (2002). Spatial separation of target and competitor cues enhances blocking of human causality judgements. Quarterly Journal of Experimental Psychology, 55B, 121-135.

Glautier, S. (2004). Asymmetry of generalization decrement in causal learning. Quarterly Journal of Experimental Psychology, 57B, 315329.

GlaUtier, S. (2008). The elemental-configural distinction: A problem of two dimensions: Commentary on Melchers, Shanks, and Lachnit (2007). Behavioural Processes, 77, 431-433.

González, F., Quinn, J. J., \& FAnselow, M. S. (2003). Differential effects of adding and removing components of a context on the generalization of conditional freezing. Journal of Experimental Psychology: Animal Behavior Processes, 29, 78-83.

HARRIS, J. A. (2006). Elemental representations of stimuli in associative learning. Psychological Review, 113, 584-605.

Lachnit, H., Schultheis, H., KöNIG, S., ÜnGÖr, M., \& Melchers, K. G. (2008). Comparing elemental and configural associative theories in human causal learning: A case for attention. Journal of Experimental Psychology: Animal Behavior Processes, 34, 303-313.

Lachnit, H., Thorwart, A., \& Schultheis, H. (2008). Comparing elemental and configural associative theories in human Pavlovian conditioning with indicators of early and late CS processing. Unpublished manuscript.

Liljeholm, M., \& BALleine, B. (2008). It's elemental my dear Watson. Behavioural Processes, 77, 434-436.
Livesey, E. J., \& Boakes, R. A. (2004). Outcome additivity, elemental processing and blocking in human causality judgements. Quarterly Journal of Experimental Psychology, 57B, 361-379.

Livesey, E. J., \& Harris, J. A. (2008). What are flexible representations? Commentary on Melchers, Shanks and Lachnit. Behavioural Processes, 77, 437-439.

Melchers, K. G., Shanks, D. R., \& Lachnit, H. (2008). Stimulus coding in human associative learning: Flexible representations of parts and wholes. Behavioural Processes, 77, 413-427.

Palmer, S. E. (1999). Vision science: Photons to phenomenology. Cambridge, MA: MIT Press.

Pavlov, I. P. (1927). Conditioned reflexes: An investigation of the physiological activity of the cerebral cortex (G. V. Anrep, Trans. \& Ed.). London: Oxford University Press.

Pearce, J. M. (1987). A model for stimulus generalization in Pavlovian conditioning. Psychological Review, 94, 61-73.

Pearce, J. M. (1994). Similarity and discrimination: A selective review and a connectionist model. Psychological Review, 101, 587-607.

Pineño, O., Denniston, J. C., Beckers, T., Matute, H., \& Miller, R. R. (2005). Contrasting predictive and causal values of predictors and of causes. Learning \& Behavior, 33, 184-196.

Schultheis, H., Thorwart, A., \& Lachnit, H. (2008a). HMS: A MATLAB simulator of the Harris model of associative learning. Behavior Research Methods, 40, 442-449.

Schultheis, H., Thorwart, A., \& Lachnit, H. (2008b). Rapid-REM: A MATLAB simulator of the replaced-elements model. Behavior Research Methods, 40, 435-441.

Shanks, D. R., Lachnit, H., \& Melchers, K. G. (2008). Representational flexibility and the challenge to elemental theories of learning: Response to commentaries. Behavioural Processes, 77, 451-453.

Thorwart, A., Schultheis, H., König, S., \& Lachnit, H. (in press). ALTSim: A MATLAB simulator for current associative learning theories. Behavior Research Methods.

WaGner, A. R. (2003). Context-sensitive elemental theory. Quarterly Journal of Experimental Psychology, 56B, 7-29.

Wheeler, D. S., Amundson, J. C., \& Miller, R. R. (2006). Generalization decrement in human contingency learning. Quarterly Journal of Experimental Psychology, 59, 1212-1223.

\section{NOTES}

1. To avoid misunderstandings, the term component is used for distinct parts of a stimulus compound; for example, a stimulus compound comprises its two components - a red light and a yellow light. Element refers to the even smaller parts of a component's representation, as hypothesized by the elemental models.

2. The REM is sometimes labeled as a "hybrid" model (Glautier, 2008; Liljeholm \& Balleine, 2008; Livesey \& Harris, 2008). However, because learning still occurs about elements and not about whole configurations, we classify the REM as a purely elemental theory. In contrast, Pearce's (1994) model assumes that the stimulus configuration as a whole gets associated. It is this kind of conditioning we refer to as configural processing in the present article.

(Manuscript received July 25, 2008; revision accepted for publication September 11, 2008.) 